\title{
Assessment of a two-dimensional hybrid-direct kinetic simulation of a Hall thruster
}

\author{
Astrid L. Raisanen* \\ University of Michigan, Ann Arbor, MI 48109 \\ Kentaro Hara ${ }^{\dagger}$ \\ Texas A\&M University, College Station, TX 77843 \\ and Iain D. Boyd ${ }^{\ddagger}$ \\ University of Michigan, Ann Arbor, MI 48109
}

\begin{abstract}
Deterministic, direct kinetic models are of interest in the simulation of plasma physics in a Hall thruster because they lack the statistical noise that exists in particle methods. The present paper is the second part of a study concerned with the evaluation of a two-dimensional, hybrid-direct kinetic (DK) model through comparison with a hybridParticle-in-Cell (PIC) model. Both models utilize quasi one-dimensional electron fluid algorithms. While the first part of this study showed a qualitative comparison between the two kinetic models, numerical thresholds, kinetic boundary conditions, and sensitivities to the electron model rendered a quantitative comparison ineffective. This work completes the benchmarking process, showing that the start up behavior of a thruster from a specified initial condition is very similar for the two simulations, provided that the hybrid-PIC model has a large number of macroparticles and a sufficiently small time step. The similarity in results implies that the DK numerical algorithm is performing properly. This work also suggests that a dynamically changing Hall thruster simulation must treat the electron mobility in a dynamic manner. Furthermore, the kinetic algorithms are sensitive to near-anode boundary conditions, and the sensitivity of the PIC simulation to numerical parameters should be carefully examined.
\end{abstract}

\section{Nomenclature}

$f \quad$ Velocity distribution function (VDF)

$t \quad$ Time, s

$n_{s} \quad$ Number density of species $\mathrm{s}, \mathrm{m}^{-3}$

$\vec{v} \quad$ Velocity, $\mathrm{m} / \mathrm{s}$

$\vec{z} \quad$ Physical space, $\mathrm{m}$

$e \quad$ Elementary charge, $\mathrm{C}$

$\vec{E} \quad$ Electric field, T

$m \quad$ Mass, $\mathrm{kg}$

$S \quad$ Collision term

$\lambda \quad$ Magnetic streamline

$\phi^{*}(\lambda)$ Thermalized potential, $\mathrm{V}$

$\phi \quad$ Potential, V

$k_{b} \quad$ Boltzmann's constant, $1.38 \times 10^{23} \mathrm{~J} / \mathrm{K}$

$T_{e} \quad$ Electron temperature, $\mathrm{K}$

$\dot{n}_{c} \quad$ Collision rate of process $\mathrm{c}, \mathrm{s}^{-1}$

$\epsilon \quad$ Electron mean energy, eV

*Ph.D. Candidate, Department of Aerospace Engineering, Student AIAA Member

${ }^{\dagger}$ Assistant Professor, Department of Aerospace Engineering, AIAA Member

$\ddagger$ James E. Knott Professor, Department of Aerospace Engineering, AIAA Fellow 


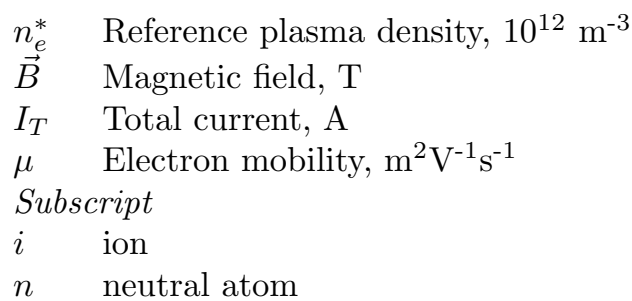

\section{Introduction}

Since Fife developed HPHall in the 1990s, two-dimensional, axisymmetric, hybrid-Particle-in-Cell (PIC) algorithms have been used to model the discharge plasma in Hall thrusters. ${ }^{1}$ Several hybrid simulation methods incorporate a fluid electron model in conjunction with a PIC kinetic model to emulate the evolution of plasma in the simulation domain. ${ }^{1-4}$ In order to provide accurate macroscopic thruster properties, hybrid-PIC methods require variable Bohm coefficients in the plasma discharge region to account for anomalous electron transport. To quantify the contribution that electron transport has on dynamically changing oscillations seen during Hall thruster operation, PIC-related statistical noise in the kinetic algorithm must be reduced. ${ }^{5}$ The first part of this study showed that DK results evolve smoothly in time and are free from statistical noise. ${ }^{6}$ The objectives of the present work are twofold: 1) to perform benchmarking of a hybrid-DK simulation with a hybrid-PIC simulation, and 2) to demonstrate the importance of the electron model and its role in the production of plasma oscillations.

In this study, the computational method for both the DK and electron algorithms are described in Section II. Some emphasis is placed on kinetic boundary conditions and electron model limitations. Section III compares and contrasts both instantaneous and time-averaged hybrid-DK and hybrid-PIC results from a case selected for the benchmarking process. This section also discusses the computational cost for both cases. In Section IV, the DK benchmarking case is compared to two other hybrid-DK cases, one which uses an electron $\lambda$ grid prior to correction, and the other which installs an upper bound for the potential in the domain. Section V compares the PIC benchmarking case with additional hybrid-PIC cases that have either fewer macroparticles or a larger time step. Finally, Section VI concludes the study and offers input regarding future work for both the hybrid-DK and hybrid-PIC simulations.

\section{Computational Method}

In the present work, the discharge plasma in a UM/AFRL P5 Hall thruster is modeled using twodimensional, axisymmetric hybrid-DK and hybrid-PIC simulations. The P5 Hall thruster, developed by the Air Force Research Laboratory and the University of Michigan for basic physics research, has been well characterized experimentally. ${ }^{7,8}$ However, this thruster is chosen for the current study because it was the focus of Koo and Boyd's previous computational work..$^{9}$

The DK and PIC simulations are used to model ions and neutral atoms, and a fluid model solves the equations of mass, momentum, and energy for electrons. In the PIC model, developed by Koo and Boyd, ions and neutral atoms are modeled using a PIC technique that simulates the motion of macroparticles via a second order, classical leapfrog scheme. Neutral atoms are depleted due to ground state ionization, which is calculated using a Monte Carlo Collision (MCC) model. ${ }^{2,9}$

\section{II.A. Direct Kinetic Method}

The DK computational method, discussed in detail in Ref. [6], is a modified, two-dimensional, axisymmetric version of the grid-based kinetic model developed by Hara et al. ${ }^{5}$ The two-dimensional transport of ions is described by the Boltzmann equation, in which the acceleration term is due to the force from the electric field, $\vec{E}$ :

$$
\frac{\partial f_{i}}{\partial t}+v_{z} \frac{\partial f_{i}}{\partial z}+v_{r} \frac{\partial f_{i}}{\partial r}+\frac{e E_{z}}{m_{i}} \frac{\partial f_{i}}{\partial z}+\frac{e E_{r}}{m_{i}} \frac{\partial f_{i}}{\partial r}=S_{i}
$$

where $e$ is the elementary charge, $f_{i}$ is the ion distribution function (VDF), $m_{i}$ is the ion mass, $v$ is the velocity term, with velocity subscripts indicating either the radial or axial component, and $S_{i}$ is the ion 
collision term. Neutral atoms do not experience any external force from the electric field, so their transport is described by Eq. (2), where the subscript $n$ denotes the species:

$$
\frac{\partial f_{n}}{\partial t}+v_{z} \frac{\partial f_{n}}{\partial z}+v_{r} \frac{\partial f_{n}}{\partial r}=S_{n}
$$

The collision terms in the Boltzmann equations are calculated as a sum of collisions due to singly charged ionization. For each ionization event, a neutral atom is deleted, and an ion with the identical velocity and physical location is created, conserving the total number of particles in the system.

$$
\begin{gathered}
S_{i}(\vec{v}, \vec{z}, t)=\dot{n}_{\text {ion }} \hat{f}_{n}(\vec{v}) \\
S_{n}(\vec{v}, \vec{z}, t)=-\dot{n}_{\text {ion }} \hat{f}_{n}(\vec{v})
\end{gathered}
$$

In Eqs. (3) and (4), $\dot{n}_{i o n}$ represents the ionization rate. Assuming that more massive neutral particles move much more slowly than electrons, the ionization rate can be approximated as a function of the mean electron energy: ${ }^{5}$

$$
\dot{n}_{i o n}=n_{n} n_{e} \zeta(\epsilon)
$$

where $n_{e}$ is the electron number density and $\epsilon$ is the electron mean energy. Note that quasineutrality dictates that $n_{e}=n_{i}$. Electron energy-dependent cross sections are obtained from Puech and Mizzi. ${ }^{10}$

In the DK method, the physical and velocity updates are split, and time integration for the solution of the collisionless Boltzmann, or Vlasov, equation is performed using Strang's time splitting technique. A finite volume method with a modified Arora-Roe limiter, discussed more in the following subsection, is used to calculate the flux terms $\left(v_{z} \frac{\partial f}{\partial z}, v_{r} \frac{\partial f}{\partial r}, a_{z} \frac{\partial f}{\partial v_{z}}\right.$, and $\left.a_{r} \frac{\partial f}{\partial v_{r}}\right) .{ }^{5}$ For numerical stability, the time step is restricted by the Courant Friedrich-Lewy (CFL) condition.

\section{II.A.1. Kinetic Boundary Conditions}

Based on the fact that excess ionization was observed at the pole pieces in the first part of this study, the updated DK boundary conditions are enumerated upon here in some detail. This section discusses the implementation of the numerical flux algorithm, particularly at the boundaries of the domain.

Within the DK simulation domain, there are four types of boundaries in physical space: outflow only; outflow with allowance of inflow due to a nonzero background pressure; zero net flux at the thruster centerline; and wall boundaries at which neutral atoms are diffusely reflected, and ions recombine to diffusely reflect as neutral atoms. The different boundary conditions are labeled in Figure 1.

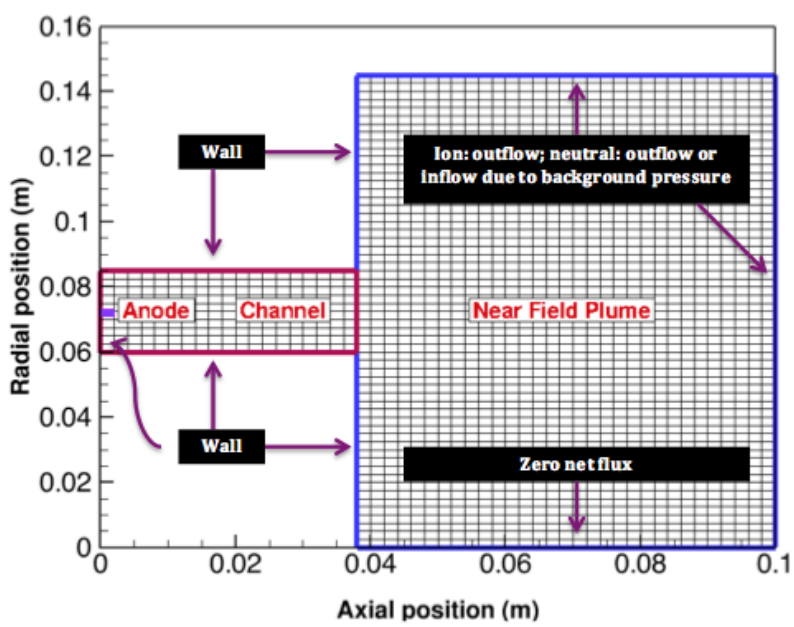

Figure 1. Simulation domain with kinetic boundary conditions.

To calculate the flux through a cell interface, the monotonic upwind scheme for conservation laws (MUSCL) is used. A modified, positivity-preserving Arora-Roe nonlinear limiter, $\Psi(r)$, acts to limit numerical extrema including undershoot and overshoot. ${ }^{11}$ The slope factor, $r$, is a function of velocity space and is determined by the distribution functions in three adjacent cells. In the second order, finite volume 
scheme, if $\Psi(r)>0.0$, then the flux through a cell interface utilizes information from three adjacent cells. Otherwise, the flux calculation uses information from only two cells at either side of the interface. At all boundaries, there are two ghost cells outside of the domain, and zeroth order extrapolation is used to control the flux through the boundary interface.

For the outflow condition, a portion of the VDF in the boundary cell is extrapolated to the adjacent ghost cells, and the other portion is set to zero. For example, at the right hand side of the domain in Figure 1 , the VDF for positive velocity space $\left(v_{z}>0\right)$ is extrapolated to the ghost cells so that ions with a positive velocity exit the domain. For negative velocity space, the VDF is set to zero in the ghost cells, and no ions can re-enter the simulation domain. In the present simulation, the thruster is assumed to expand into a perfect vacuum. However, in the case of a nonzero background pressure, neutral particles with an effective density based on the pressure are allowed to re-enter the domain as a half Maxwellian at the background temperature. At the thruster centerline, the net flux through the interface is zero. This requires a coordinate transform in the boundary cell to mirror the outgoing velocity. In the case of recombination and/or diffuse reflection at the walls, the primary challenge is to conserve the total number of particles in the system. This is accomplished by ensuring that the ion calculation is conducted first so that the VDF can be stored and used in the neutral atom calculation. The stored, average ion density is assumed to be equivalent to the recombined neutral density, which re-enters the domain as a half-Maxwellian based on the thruster wall temperature. A similar procedure is followed to model the diffuse reflection of neutral atoms.

\section{II.B. Fluid Electron Model}

The electron solver is extracted from Koo and Boyd's hybrid-PIC model, tested to ensure consistency, and integrated into the hybrid-DK framework. Because the electrons have a much smaller characteristic time scale than that for ions, they are assumed to be at steady state on the time scale of the ions. Therefore, the electrons are approximately at thermal equilibrium along magnetic field lines.

\section{II.B.1. General Electron Algorithm}

Neglecting inertial terms and collisional effects, the momentum equation parallel to the magnetic field reduces to a balance between pressure and electric forces. Integration along a magnetic field line results in the thermalized potential, $\phi^{*}(\lambda)$, which is a reduced description of the plasma potential along a magnetic field line:

$$
\phi^{*}(\lambda)=\phi-\frac{k_{b} T_{e}}{e} \ln \frac{n_{e}}{n_{e}^{*}}
$$

where $T_{e}$ is the electron temperature, $k_{b}$ is Boltzmann's constant, and $n_{e}^{*}$ is the reference plasma density. In the cases discussed herein, the reference density is constant, i.e. $n_{e}^{*}=1.0 \times 10^{12} \mathrm{~m}^{-3}$. $\lambda$ is a magnetic field stream function, and the $\lambda$ streamlines act as the electron grid. Figure 2 shows the electron $\lambda$ grid for the present study. In general, the superscript $*$ indicates a reference property for a $\lambda$-line. Eq. (6) is used to calculate the two-dimensional potential, $\phi$ in the domain, from which the electric field can be evaluated. $\phi^{*}(\lambda)$ is obtained via the one-dimensional Ohm's Law $(J=\sigma E)$ and current conservation perpendicular to the magnetic field.

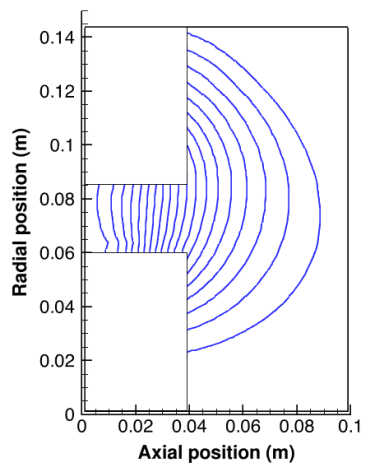

Figure 2. $\lambda$ grid for the P5 Hall thruster. 


\section{II.B.2. Model Limitations}

In order to complete this study, possible shortcomings related to the electron model are studied closely. Most of the findings are not discussed in detail here because they are not relevant to the benchmarking case. However, it becomes clear that the kinetic algorithm is very sensitive to the electron algorithm outputs, but additional accuracy in the electron model renders the simplified boundary conditions at the anode and cathode $\lambda$-lines insufficient and the solution becomes unstable. Therefore, this study attempts to utilize the electron model in a nearly unaltered state; threshold limits are adjusted, if necessary, but the algorithm and boundary conditions remain the same.

In order to calculate the total current in the system, the potential at the anode and cathode must be known. In this model, $\phi^{*}$ is assumed to float at the anode and cathode $\lambda$-lines, and it is calculated with the assumption that $\phi$ is fixed at those locations. The current in the system is conserved, and the following expression for its value, $I_{T}$, is obtained after simplification:

$$
I_{T}=\frac{\phi^{*}\left(\lambda_{a}\right)-\phi^{*}\left(\lambda_{c}\right)}{d \lambda} \sum_{\lambda_{a}}^{\lambda_{c}} \int_{S} e n_{e} \mu r B \partial S-\sum_{\lambda_{a}}^{\lambda_{c}} \int_{S} e n_{e} \mu r B\left[\ln \left(\frac{n_{e}}{n_{e}^{*}}\right)-1\right] \frac{k_{b}}{e} \frac{\partial T_{e}}{\partial \lambda} \partial S+\sum_{\lambda_{a}}^{\lambda_{c}} \int_{S} e n_{i} v_{i} \partial S
$$

For the two-dimensional solution of the potential, Dirichlet boundary conditions are applied at the left and right hand sides of the domain, which do not correspond to the anode and cathode $\lambda$-lines. Since the domain of interest lies between the anode and cathode $\lambda$-lines, and furthermore, since the potential should not change much outside of this region, this is a reasonable approach. However, as a result, the kinetic models exhibit unusual behavior at the centerline of the thruster channel, just upstream of the anode $\lambda$-line. This is explained in more detail in Section III.

In addition, it seems unlikely that the reference plasma density, $n_{e}^{*}$, is best described by a single value for the entire domain. One alternative is to select the average value of $n_{e}$ for each $\lambda$-line. However, this method renders the potential solution outside of the active domain more variable, so it is left for future studies.

\section{II.B.3. Numerical Constraints and Issues}

Particularly for the PIC case, it is important to ensure that there is a minimum threshold plasma and neutral density in case a single cell is devoid of macroparticles over the course of a single time step. The DK simulation does not have this same restriction, but it is possible to utilize the DK model to determine reasonable threshold values via instantaneous results. For both the DK and PIC simulations, the maximum potential threshold is removed to allow for additional plasma evolution in the channel. Minimum threshold values are applied for $n_{i}, n_{n}$, and $\phi$.

Koo and Boyd's electron simulation is also updated to include a corrected $\lambda$-grid. The updated $\lambda$-grid corrects an unintended perturbation to the evolution of the plasma properties, particularly inside the channel. This correction allows for a damped solution of the discharge current in the case where the electron mobility profile is fixed. This is discussed more in Section III.

\section{II.C. Electron Mobility Model}

In this study, a static profile from Koo and Boyd's work is used to model the electron mobility in the Hall thruster domain. ${ }^{9}$ The axially-varying, radially constant mobility profile is constructed from a combination of experimental data and modeling assumptions. The primary assumption is that the electron current density can be estimated at the thruster channel centerline. Figure 3 shows the best fit mobility profile used in this work, compared to both a classical mobility profile and empirical results. ${ }^{2}$ 


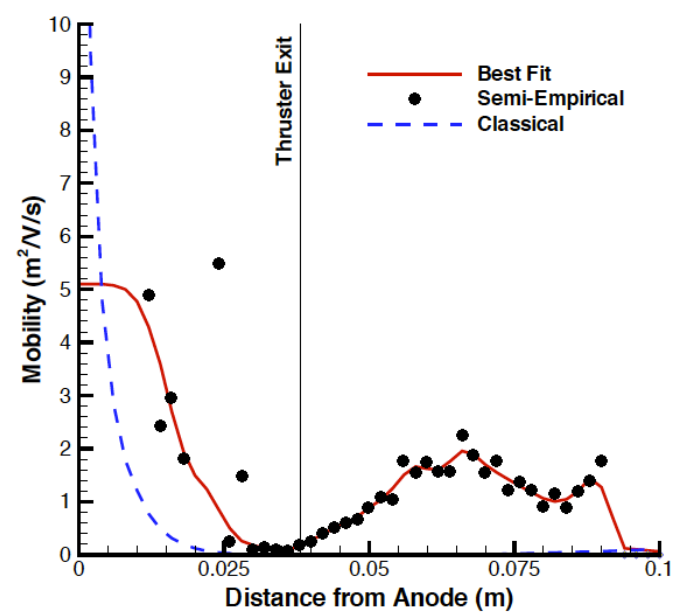

Figure 3. Static mobility profile for UM/AFRL P5-Hall thruster. ${ }^{2}$

\section{II.D. DK Interpolation to $\lambda$-lines}

Since the hybrid-PIC simulation tracks small macroparticles in physical space, it is straightforward to determine where the particles reside with respect to the $\lambda$-lines that they cross. Since the DK simulation is grid-based, it is necessary to interpolate information from the Cartesian cells to the $\lambda$ grid and vice-versa. If a $\lambda$-line cuts a Cartesian cell in two, the average number density in the DK Cartesian cell can be fractionally split, if necessary, so that it is appropriately distributed to the $\lambda$ grid.

\section{Benchmarking Setup and Results}

Certain input values, threshold parameters, and the grid discretization for the hybrid-DK and hybridPIC simulations are shown in Table 1. Note that the average macroparticle count for the PIC simulation is calculated based on the assumption that the maximum allowable number of macroparticles fill up the domain.

\section{III.A. Boundary Conditions}

In both simulations, incoming neutral atoms are emitted from an annular opening with a height of $0.2 \mathrm{~mm}$ located at the anode side of the thruster, centered at $[\mathrm{r}=0.0725 \mathrm{~m}]$. Assuming a neutral number density of $1.0 \times 10^{20} \mathrm{~m}^{-3}$, the Knudsen number is greater than 20, and the inlet neutral flow can be assumed to be in the free molecular regime. Thus, the velocity of the injected particles is described by a half Maxwellian velocity distribution biased with axial velocity. The assumed anode reservoir temperature is $700 \mathrm{~K}$. Both simulations consider diffuse reflections at the walls, and ions recombine to atoms when they hit the thruster walls or pole pieces. The channel chamber temperature is assumed to be $700 \mathrm{~K}$.

Although the electron equations are solved between the anode $\lambda$-line and the cathode $\lambda$-line, ionization is allowed everywhere in the domain. Upstream of the anode $\lambda$-line, the energy has a Dirichlet boundary condition of $1.0 \mathrm{eV}$. Downstream of the cathode $\lambda$-line, a Dirichlet boundary condition of $1.0 \mathrm{eV}$ is also applied. The potential is set to $275 \mathrm{~V}$ and $0 \mathrm{~V}$ on the left and right hand sides of the domain, respectively. In this simulation, the potential is not allowed to drop below $0 \mathrm{~V}$ anywhere in the domain.

The initial condition for the number density in the domain is identical to that applied in Koo's previous work. ${ }^{2}$ Initially, a uniform neutral atom density of $n_{n}=1.0 \times 10^{20} \mathrm{~m}^{-3}$ is applied inside the channel, and $n_{n}=1.0 \times 10^{18} \mathrm{~m}^{-3}$ in the plume. A uniform ion density of $n_{i}=5.0 \times 10^{15} \mathrm{~m}^{-3}$ is applied inside the channel, and $n_{i}=1.0 \times 10^{15} \mathrm{~m}^{-3}$ in the plume. Velocities in the hybrid-DK domain are initially given a normalized Maxwellian distribution in each cell corresponding to a temperature of $700 \mathrm{~K}$. In the hybrid-PIC simulation, thermal velocities for both ions and neutral atoms are initially assigned by random sampling from a Maxwellian VDF corresponding to a neutral background temperature of $1000 \mathrm{~K}$. In the future, this difference between the two simulations should be rectified. 
Table 1. Hybrid-DK Simulation Parameters

\begin{tabular}{|c|c|c|c|}
\hline Parameter & Description & Hybrid-DK & Hybrid-PIC \\
\hline Channel length & $L_{c h}$ & $38 \mathrm{~mm}$ & $38 \mathrm{~mm}$ \\
\hline Channel diameter & $D_{c h}$ & $25 \mathrm{~mm}$ & $25 \mathrm{~mm}$ \\
\hline Axial domain length & $L_{z}$ & $100 \mathrm{~mm}$ & $100 \mathrm{~mm}$ \\
\hline Radial (plume) domain length & $L_{r}$ & $145 \mathrm{~mm}$ & $145 \mathrm{~mm}$ \\
\hline Neutral atom axial velocity space & {$\left[v_{z, \min }, v_{z, \max }\right]$} & {$[-1200 \mathrm{~m} / \mathrm{s}, 1500 \mathrm{~m} / \mathrm{s}]$} & $N / A$ \\
\hline Neutral atom radial velocity space & {$\left[v_{r, \min }, v_{r, \max }\right]$} & {$[-1200 \mathrm{~m} / \mathrm{s}, 1200 \mathrm{~m} / \mathrm{s}]$} & $N / A$ \\
\hline Ion axial velocity space & {$\left[v_{z, \min }, v_{z, \max }\right]$} & {$[-16000 \mathrm{~m} / \mathrm{s}, 30000 \mathrm{~m} / \mathrm{s}]$} & $N / A$ \\
\hline Ion radial velocity space & {$\left[v_{r, \min }, v_{r, \max }\right]$} & {$[-14000 \mathrm{~m} / \mathrm{s}, 14000 \mathrm{~m} / \mathrm{s}]$} & $N / A$ \\
\hline Average neutral macroparticles/cell & Number & $N / A$ & 800 \\
\hline Average ion macroparticles/cell & Number & $N / A$ & 300 \\
\hline Cell size in physical space & {$[\Delta z, \Delta r]$} & {$[2.0 \mathrm{~mm}, 2.5 \mathrm{~mm}]$} & {$[2.0 \mathrm{~mm}, 2.5 \mathrm{~mm}]$} \\
\hline Bins in physical space & $N_{z, r}$ & 1988 & 1988 \\
\hline Bins in atom velocity space & $N\left(v_{z}\right), N\left(v_{r}\right)$ & 108,240 & $N / A$ \\
\hline Bins in ion velocity space & $N\left(v_{z}\right), N\left(v_{r}\right)$ & 230,140 & $N / A$ \\
\hline Number of $\lambda$-lines & $N_{\lambda}$ & 21 & 21 \\
\hline Global timestep & $\Delta t$ & $1.0 \times 10^{-8} \mathrm{~s}$ & $1.0 \times 10^{-8} \mathrm{~s}$ \\
\hline Electron timestep & $\Delta t_{\text {electron }}$ & $5.0 \times 10^{-11} \mathrm{~s}$ & $5.0 \times 10^{-11} \mathrm{~s}$ \\
\hline Minimum plasma density & $n_{i, \min }$ & $5.0 \times 10^{12} \mathrm{~m}^{3} / \mathrm{s}$ & $5.0 \times 10^{12} \mathrm{~m}^{-3}$ \\
\hline Minimum neutral density & $n_{n, \min }$ & $1.0 \times 10^{15} \mathrm{~m}^{3} / \mathrm{s}$ & $1.0 \times 10^{15} \mathrm{~m}^{-3}$ \\
\hline Anode potential & $\phi_{\text {anode }}$ & $275 \mathrm{~V}$ & $275 \mathrm{~V}$ \\
\hline Anode mass flow rate & $\dot{m}$ & $10.2 \mathrm{mg} / \mathrm{s}$ & $10.2 \mathrm{mg} / \mathrm{s}$ \\
\hline
\end{tabular}




\section{III.B. Benchmarking Results}

The discharge current from the PIC and DK based simulations is shown in Figure 4a. The low amplitude current that is seen after startup from $t=0.20 \mathrm{~ms}$ to $t=0.95 \mathrm{~ms}$ is highlighted in Figure $4 \mathrm{~b}$.

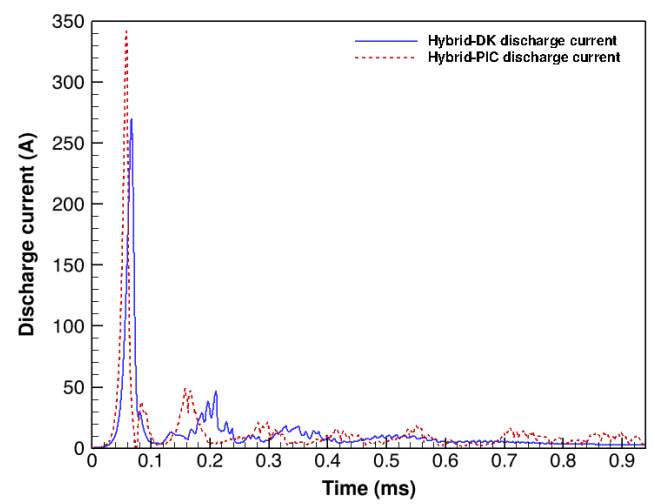

(a) Startup discharge current vs time.

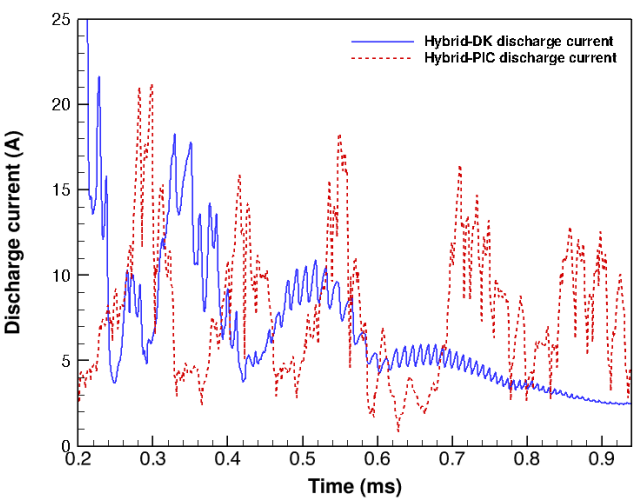

(b) Discharge current vs time, zoomed in.

Figure 4. Discharge current vs time.

Although the results are not identical, it is clear that both the amplitude and frequency of the discharge current are similar, particularly at start-up. It appears that the hybrid-PIC current is slightly shifted in time from the hybrid-DK current. In the DK case, the current first exhibits a high frequency oscillation around $120 \mathrm{kHz}$ and then damps to approximately $3.5 \mathrm{~A}$ around $2.0 \mathrm{~ms}$ (not shown). There is not sufficient data to support this statement for the hybrid-PIC case, but behavior thus far indicates that it will retain a slight oscillation. While the behavior of the discharge current is not comparable to experimental results, a damped solution is not necessarily unexpected, since this case has a fixed electron mobility profile. The previous work of Hara et al. suggests that a low frequency breathing mode oscillation is the result of a perturbation in electron energy due to electron transport. ${ }^{12}$ A fixed mobility profile should not allow for such an energy perturbation. However, this result requires additional study to ensure that it is not coupled with the behavior of the plasma near the anode $\lambda$-line, discussed in Subsection III.B.3, due to an insufficient anode boundary condition.

Since the present simulation does not emulate experimental results, it does not make sense to present performance parameters such as thrust and specific impulse. However, instantaneous and time-averaged plasma properties can be compared as part of the benchmarking process.

\section{III.B.1. Instantaneous Results}

Instantaneous plasma properties including the neutral and ion number densities, the ionization rate, the potential, and the electron energy are evaluated at different times, dependent on the behavior of the discharge current. The behavior at point 1 in Figure 5d corresponds to the highest point in the discharge current. For this data point, the plasma density in Figure 5a is high in both the channel and plume, as it has not yet been flushed out of the domain. The PIC current and density are both slightly higher than the comparable DK values. The ionization rate in Figure $5 \mathrm{~b}$ as well as the electron energy and potential in Figure 5c are nearly identical for both the PIC and DK simulations. Note that the potential has a minimum bound of 0 V. 


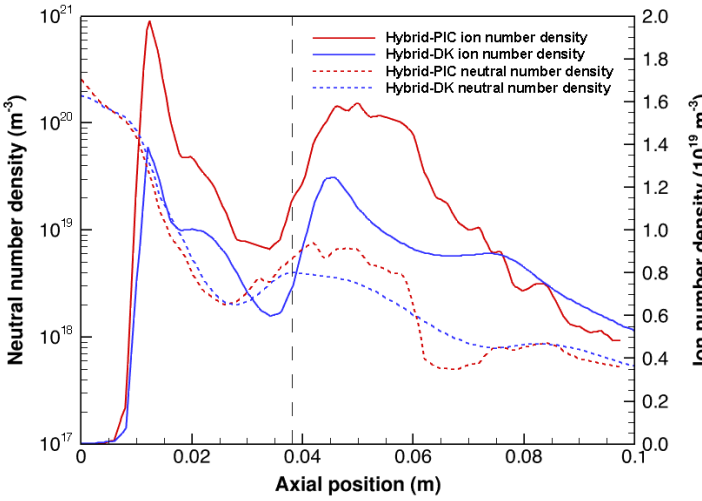

(a) Number density.

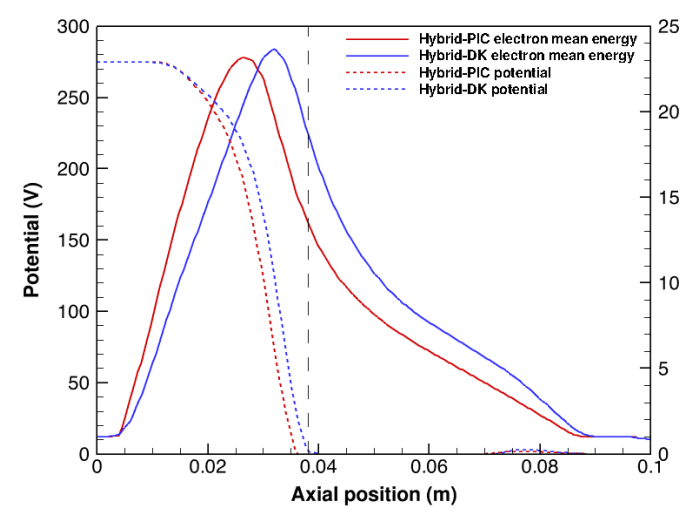

(c) Electron energy and potential.

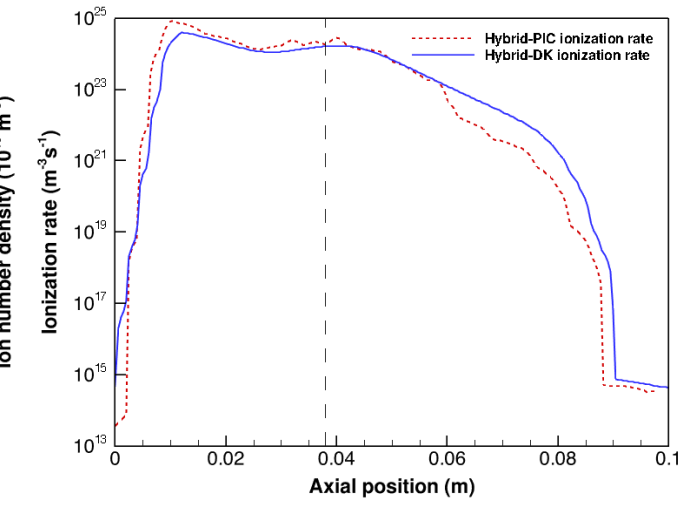

(b) Ionization rate.

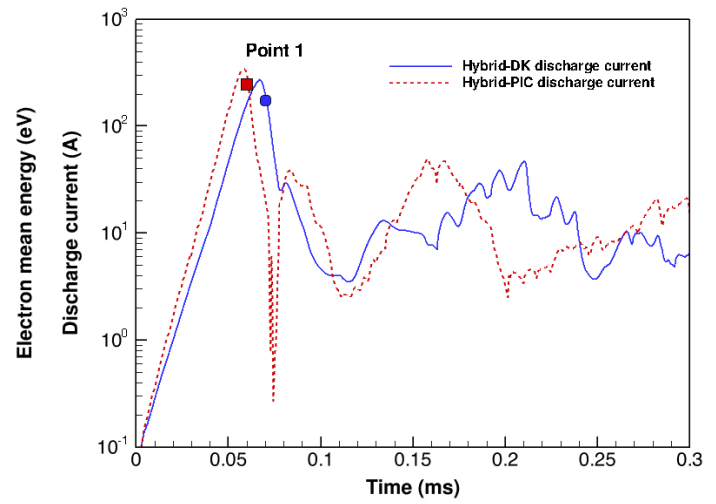

(d) Discharge current vs time at point 1.

Figure 5. Axial profiles of instantaneous properties along the channel centerline at point 1.

The behavior at point 2 in Figure $6 \mathrm{~d}$ corresponds to a time shortly after the minimum discharge current has been reached, and plasma begins to fill the channel. For this data point, the plasma density in Figure $6 \mathrm{a}$ is high in the channel and low in the plume. However, there is an early indication that ions may build up just upstream of the anode $\lambda$-line, which is located on the channel centerline at $[z=0.011 \mathrm{~m}]$ but varies slightly in the radial direction. The ionization rate in Figure $6 \mathrm{~b}$ is slightly higher in the DK case, compared to the PIC case. At this point in time, the DK electron energy and potential in Figure 6c do not change sharply at the anode $\lambda$-line as they do for the PIC case. 


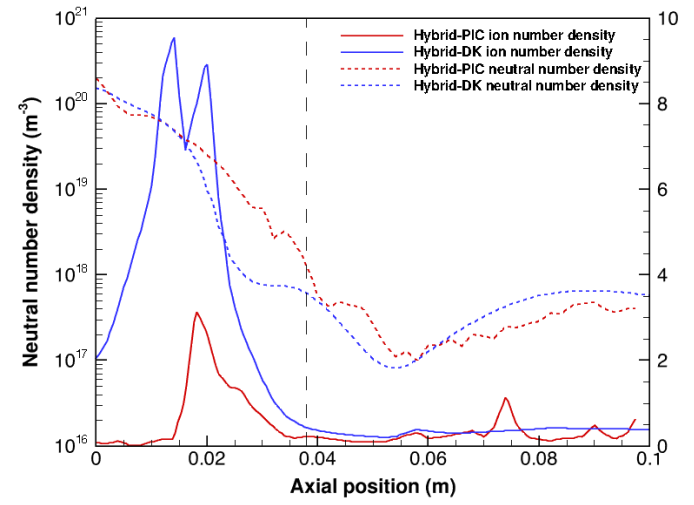

(a) Number density.

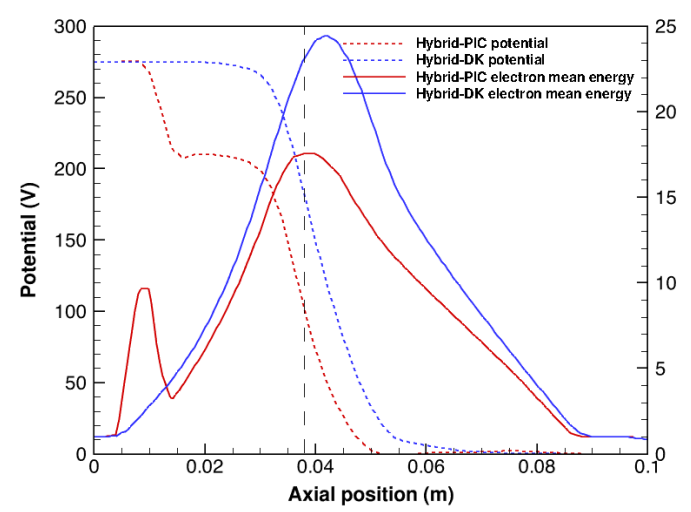

(c) Electron energy and potential.

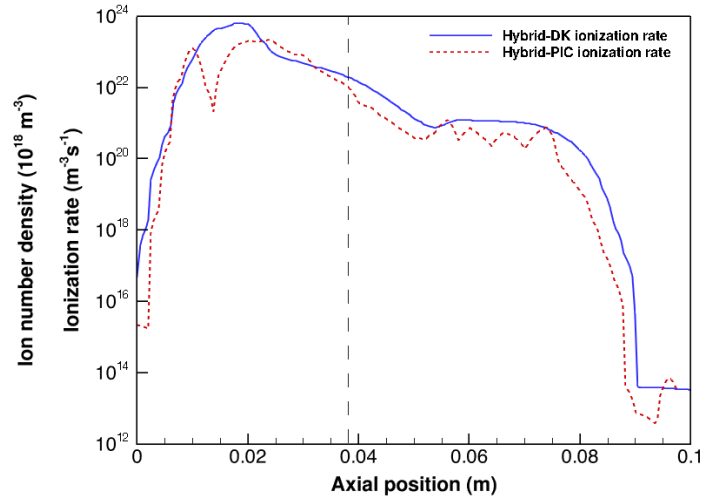

(b) Ionization rate.

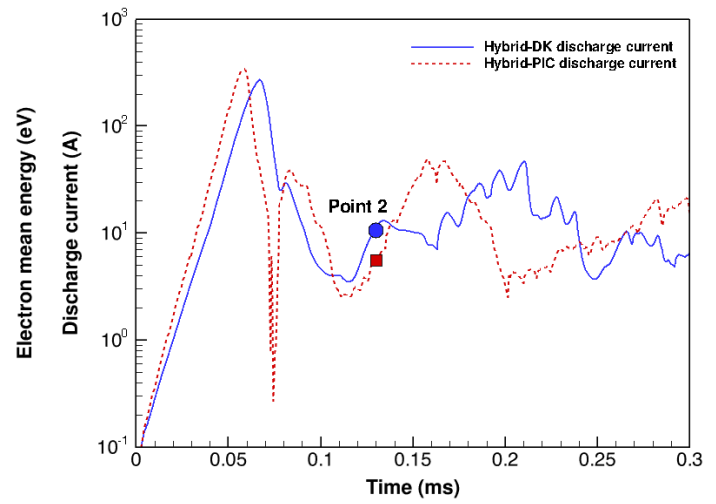

(d) Discharge current vs time at point 2.

Figure 6. Axial profiles of instantaneous properties along the channel centerline at point 2.

The behavior at point 3 in Figure $7 \mathrm{~d}$ corresponds to another relative maximum in the discharge current, as ions begin to leave the domain. For this data point, the plasma density in Figure 7a has two distinct regions in the DK simulation channel and just one in the PIC channel; both domains show a bulk of ions in the near-field plume, exiting the domain. For the DK case, there is further indication that ions may build up inside the channel near the anode $\lambda$-line. The ionization rate in Figure $7 \mathrm{~b}$ is similar in both cases, but it is slightly higher for the DK simulation just upstream of the anode $\lambda$-line. The electron energy in Figure 7c is lower for the DK case. This correlates well with the potential profile; since there are no large gradients in the potential, there are no large gradients in the energy profile. In the PIC case, the potential falls off sharply just upstream of the channel exit, and the corresponding instantaneous maximum energy is high. 


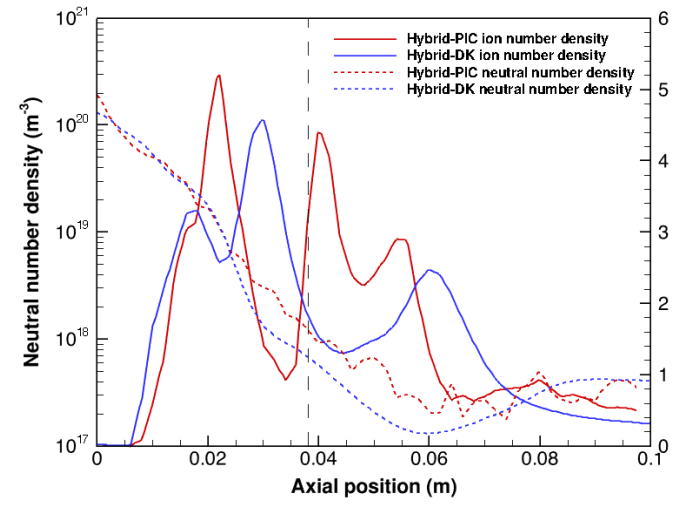

(a) Number density.

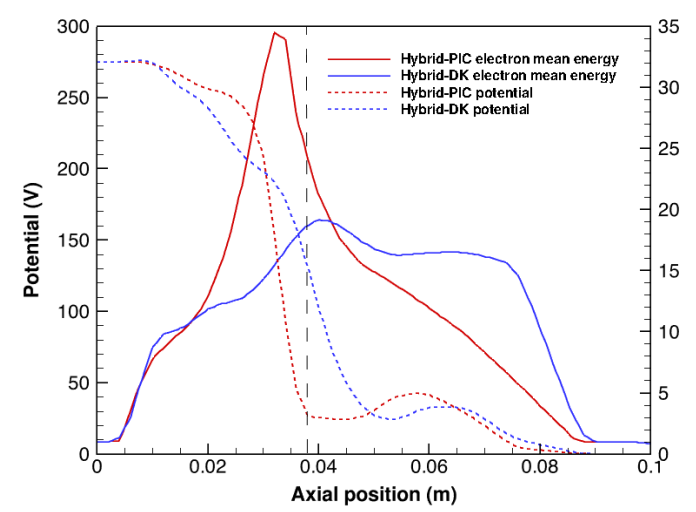

(c) Electron energy and potential.

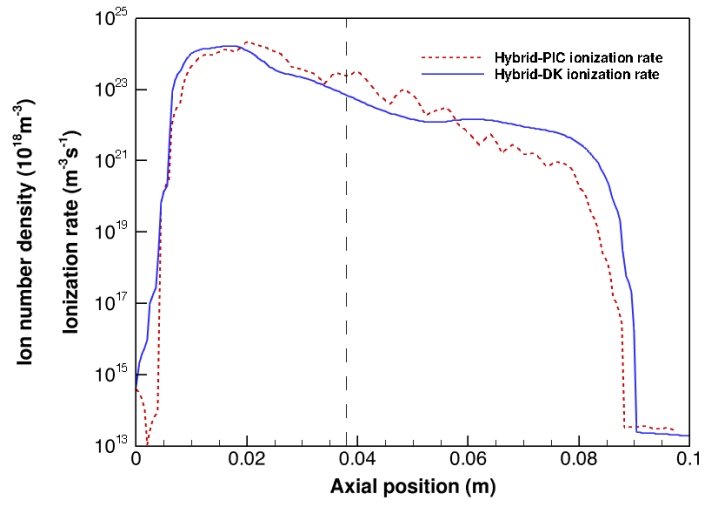

(b) Ionization rate.

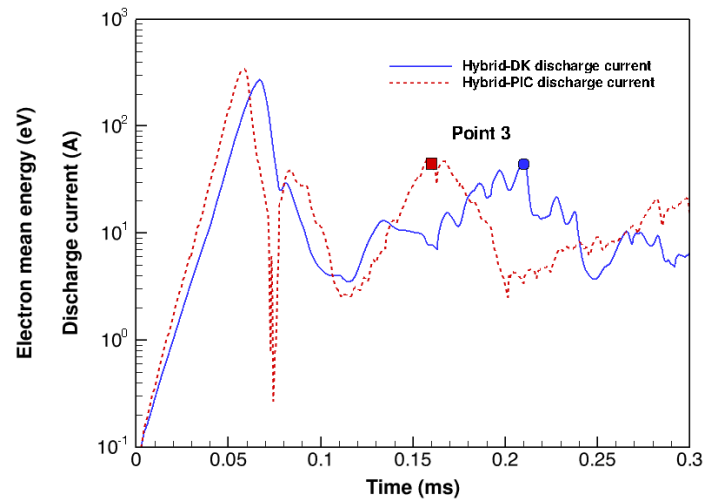

(d) Discharge current vs time at point 3.

Figure 7. Axial profiles of instantaneous properties along the channel centerline at point 3.

\section{III.B.2. Time-averaged Results}

Time-averaged results are evaluated at $\mathrm{t}=0.94 \mathrm{~ms}$ with a sampling interval of $1.0 \times 10^{-3} \mathrm{~ms}$. Results indicate that the two simulations behave quite similarly, with the exception of a difference in the ion number density and the neutral number density in Figure 8a. In the DK case, the neutral number density is higher throughout the domain, which corresponds to a slightly higher plasma density. This would seem to imply that the anode mass flow rate is higher for the DK case, but this does not appear to be the case. However, it is worth noting that the average DK density continues to decrease as the simulation progresses, as displayed in Figure 10a. As indicated in Figure 8b, the ionization rate is similar for both cases, and the average potential profile in Figure 8c is nearly identical. The energy for the DK case is slightly lower than that of the PIC case, but both exhibit relative maxima at the location of the anode $\lambda$-line. 


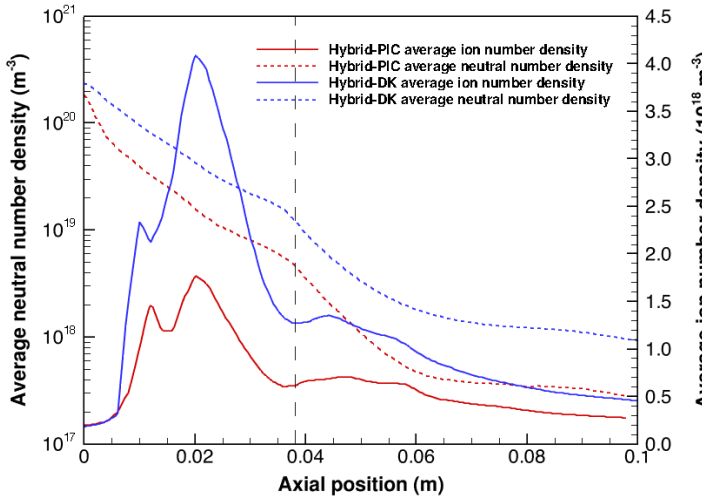

(a) Number density.

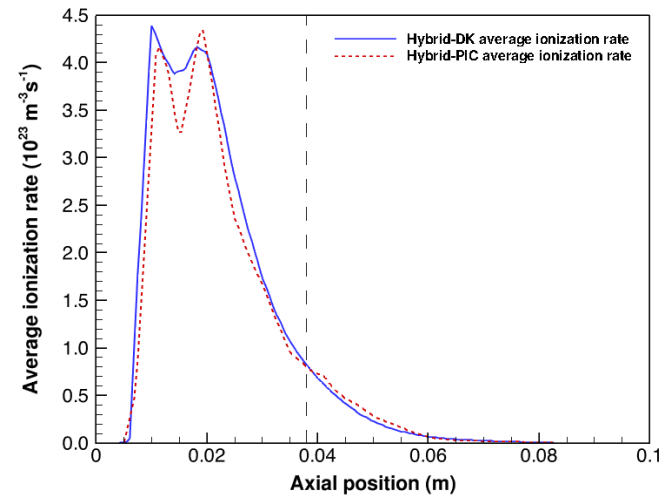

(b) Ionization rate.

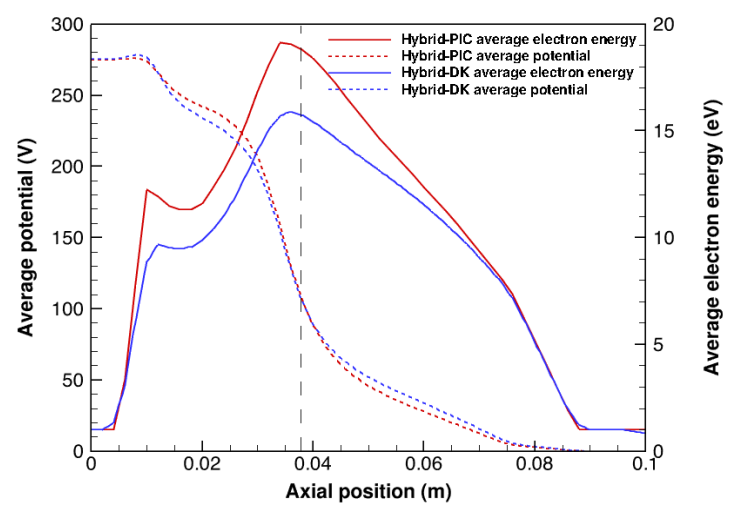

(c) Potential and electron mean energy.

Figure 8. Axial profiles of average properties along the channel centerline at $t=0.94 \mathrm{~ms}$.

\section{III.B.3. Anode boundary condition}

As indicated previously, the anode boundary condition may not be sufficient for accurate future simulations. However, in this benchmarking case, it is promising that both the hybrid-PIC and hybrid-DK simulations respond similarly to this condition. To see the impact more clearly, additional DK instantaneous results are included in this section. At $t=1.0 \mathrm{~ms}$, the instantaneous DK results show two distinct regions of ionization. Figure $9 \mathrm{a}$ is a contour plot of the instantaneous ion number density. A small region of high ion density (shown in red) is located partially upstream of the anode $\lambda$-line, which is located at $[\mathrm{z}=0.011 \mathrm{~m}]$ at the channel centerline. This small region of density in the near-anode region is clearly higher than the plasma density just upstream of the channel exit. These results do not correspond well with the average results in Figure 8a, where the plasma density near the exit is highest. This indicates that the plasma may oscillate at short time intervals. However, this oscillation may not be visible in the discharge current if the current is dampened due to the anode boundary condition.

In Figure 9b, it is clear that the ion streamlines initiate from the near-anode region, where the potential is highest. The energy contour in Figure 9c is slightly high adjacent to the anode $\lambda$-line, indicating a response to the high density plasma in the upstream region. Figure $9 \mathrm{~d}$ is an exponential plot of the neutral number density. It is clear that neutrals are not sufficiently ionized inside the channel, since there is a relatively large neutral population in the near-field plume. The oddity in the neutral density contour in the plume near the channel centerline is due to the finite discretization of velocity space. This is because, near the anode gas distributor, radial velocity components advect away from the channel centerline, and the only particles that reach the channel centerline are beam-like with zero radial velocity. ${ }^{5}$ 


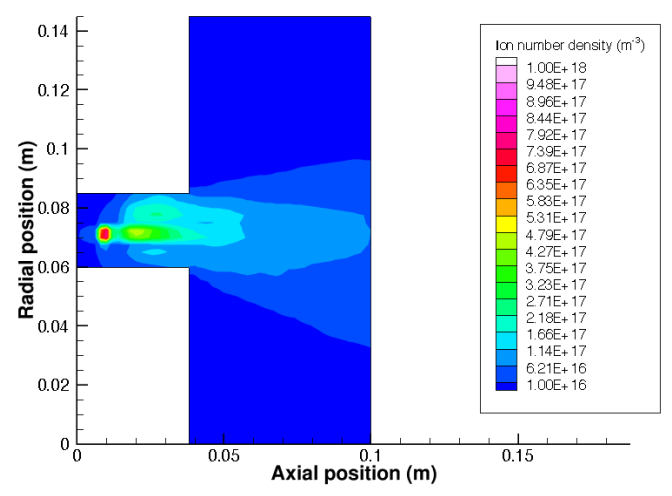

(a) Ion number density.

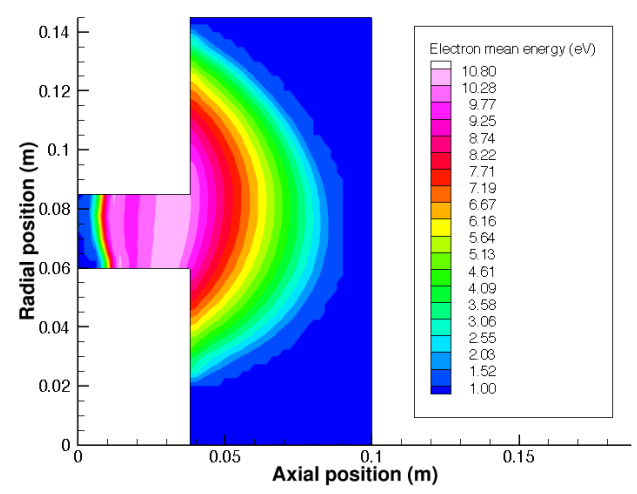

(c) Electron energy.

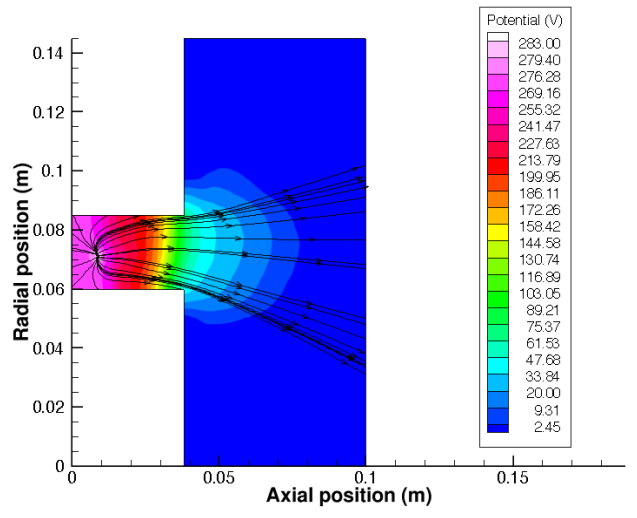

(b) Potential overlaid with ion velocity streamlines.

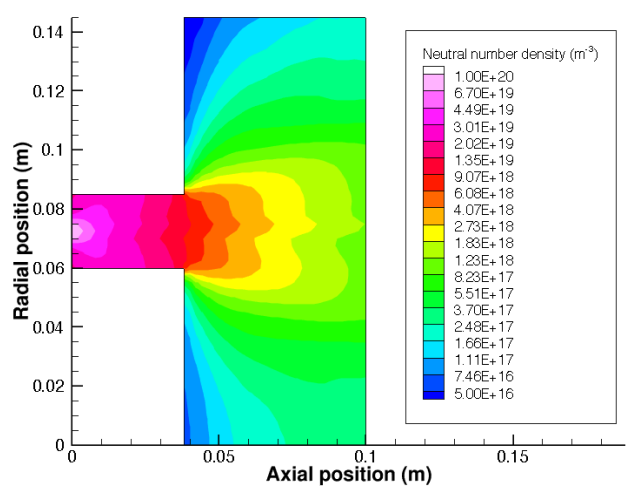

(d) Neutral number density.

Figure 9. Contours of instantaneous Hybrid-DK properties at $\mathrm{t}=1.0 \mathrm{~ms}$.

\section{III.C. Computational Cost}

For $1.0 \mathrm{~ms}$ of simulation time, the hybrid-DK simulation requires a wall time of approximately 3.3 days using 24 processors. Although the computational cost is higher for the hybrid-DK case, the serial hybridPIC simulation takes approximately 12.5 days. Since the cost for the model scales with $N_{z, r} N_{v}$, a serial DK model would be expected to take approximately 53 times longer than the PIC simulation. This estimate assumes that the DK simulation maintains $108 \times 240$ neutral velocity bins and $230 \times 140$ ion velocity bins in each physical cell. For the PIC simulation, the estimate assumes that there are 1100 macroparticles per cell, on average. Clearly, the DK simulation speed up due to parallelization is significant, and if a high macroparticle count is necessary for the PIC simulation, it should also be parallelized in the future.

\section{Additional DK Simulation Results}

Average results for the benchmarking case at the channel centerline are compared with results from Cases I and II in Figure 10a and Figure 10b. The comparison is taken at a simulation time of $2.0 \mathrm{~ms}$, and the implications of these results are explained within subsections IV.A and IV.B. These cases show that the kinetic algorithm is sensitive to threshold values and the accuracy of the electron algorithm. 


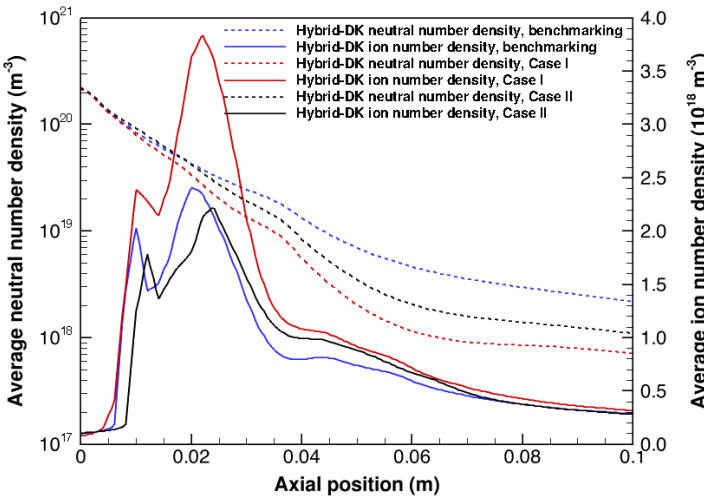

(a) Ion and neutral atom density.

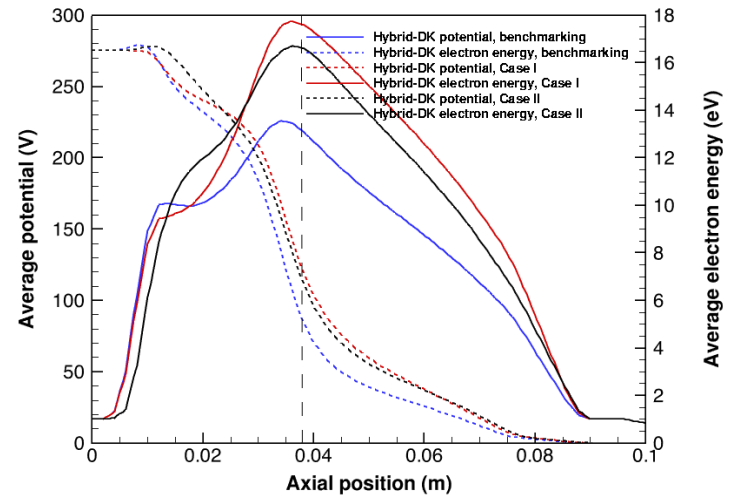

(b) Potential and electron energy.

Figure 10. Axial profiles of average Hybrid-DK properties along the channel centerline at $t$ $=2.0 \mathrm{~ms}$.

\section{IV.A. Case I: maximum threshold potential}

In this section, the maximum threshold potential is applied to the DK domain. As shown in Figure 11, if the potential is limited to a maximum of $275 \mathrm{~V}$, the resultant discharge current has a higher amplitude of approximately $6.4 \mathrm{~A}$, compared to $3.5 \mathrm{~A}$ for the benchmarking case. Additionally, the current displays a high oscillation frequency around $50.0 \mathrm{kHz}$, centered about the mean current. The average ion number density, shown in Figure 10a, is higher than that in the benchmarking case, resulting in a correspondingly lower neutral atom density in the near-field plume. In either case, there is a relative maximum ion number density at the anode $\lambda$-line. The maximum electron energy in Figure 10b is approximately $18 \mathrm{eV}$, compared to $13 \mathrm{eV}$ for the benchmarking case.

In this case, the electric field oscillates, with the peak axial value moving back and forth between the interior of the channel and the channel exit. This suggests that the high frequency oscillation may be described by the ion transit-time oscillation. This oscillation, typically in the $100-500 \mathrm{kHz}$ range, has an oscillation period on the order of the transit time of ions passing through the acceleration region, and it is associated with an oscillating electric field and the formation of a tail in the ion energy distribution function (IEDF).$^{13}$

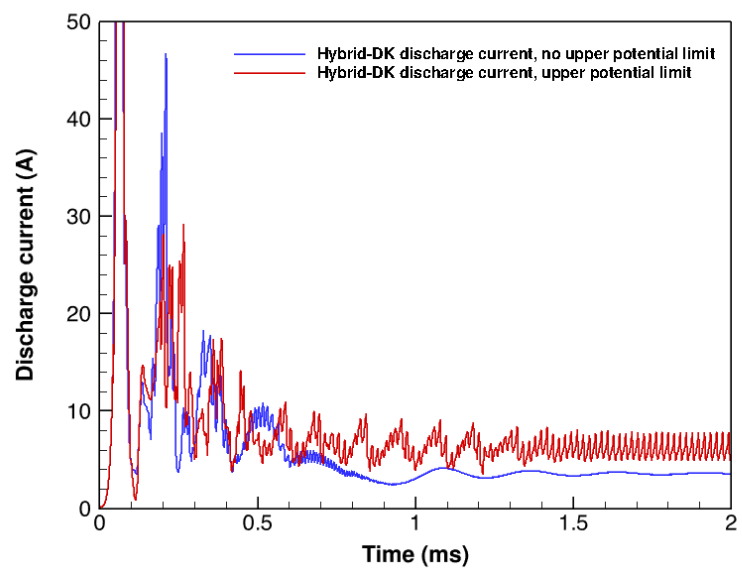

Figure 11. Case I discharge current vs time. 


\section{IV.B. Case II: original $\lambda$-grid}

Since the electron mobility profile utilized in this study is the result of experimental measurements and modeling assumptions, it is possible that its formulation is dependent on the $\lambda$ grid, or at least on results that were produced via its utilization. The results shown for Case II in Figures 10a and 10b correspond to a simulation that uses the original $\lambda$-grid, which has a slight error that shifts the location of the $\lambda$-lines. Although the change in the actual value of $\lambda$ is small, the error propagates into the calculation of the location of $\lambda$-lines for the interpolation of properties to the electron grid, particularly inside the channel. Near the anode, the axial grid location of $\lambda$ is changed by up to twenty percent. The difference is small outside of the near-anode region, and minimal in the plume. The resultant discharge current in Figure 12 exhibits a low frequency oscillation around $5 \mathrm{kHz}$. This case exhibits a high maximum electron energy near the channel exit, shown in Figure 10b. However, the corresponding ion number density in Figure 10a is relatively low.

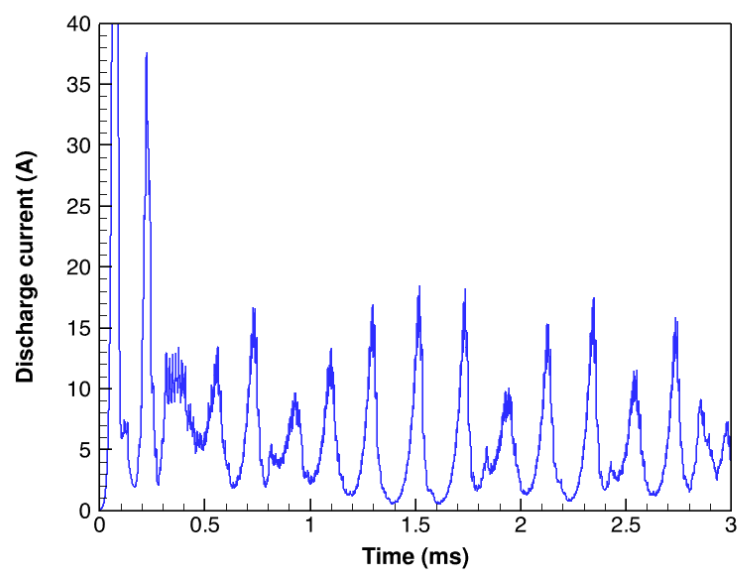

Figure 12. Case II discharge current vs time.

\section{PIC simulation convergence}

In order to determine the appropriate macroparticle count and time step for the PIC benchmarking case, several hybrid-PIC simulations are attempted with different configurations. The original case utilizes a time step in accordance with the guideline suggested in Koo and Boyd's previous work: ${ }^{2}$

$$
\Delta t=\alpha_{t} \Delta x \sqrt{\frac{m_{i}}{2 q V_{d}}}
$$

Eq. (8) approximates the time step, $\Delta t$, to be equal to the grid spacing, $\Delta x$, multiplied by the speed of a perfectly accelerated ion. $\alpha_{t}$ is a safety factor less than one to account for the possibility of ion superacceleration. Assuming that $\alpha_{t}=0.5$, then $\Delta t=7.0 \times 10^{-8} \mathrm{~s}$. The recommended number of macroparticles is approximately 30 macroparticles/cell. ${ }^{2}$ Since this study attempts to quantify statistical noise attributed to the particle algorithm, the time step is reduced, and the particle count is significantly increased in an attempt to reduce statistical noise due to the kinetic algorithm as much as possible.

Initial simulation results with a time step and particle count conforming to the requirements above result in a chaotic discharge current that does not appear useful for the benchmarking process. Thus, for consistency, a time step equal to that in the DK simulation is chosen, i.e. $\Delta t=1.0 \times 10^{-8} \mathrm{~s}$. The macroparticle count is increased to ensure that it is sufficiently matched to the time step. Figure 13 shows the discharge current for the benchmarking case (1100 particles/cell) and a second case with fewer macroparticles (700 particles/cell). Although the results are not identical, they are converging, so it is likely that the chosen particle count is sufficient for the benchmarking simulation. 


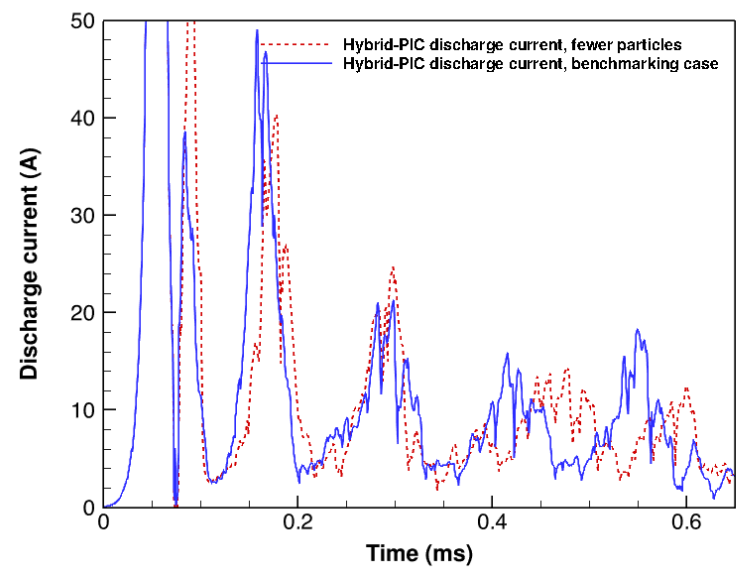

Figure 13. Discharge current vs time, different macroparticle counts.

To highlight the difficulty of simulation convergence at the level of the discharge current, Figure 14 shows results for simulations that have an average of 1100 macroparticles per cell with different time steps. The results for $\Delta t=5.0 \times 10^{-8} \mathrm{~s}$ are markedly different than the cases with $\Delta t=1.0 \times 10^{-8} \mathrm{~s}$ and $\Delta t=3.0 \times 10^{-8}$ s. The high $\Delta t$ case exhibits a strong oscillation marked by a high amplitude in the discharge current.

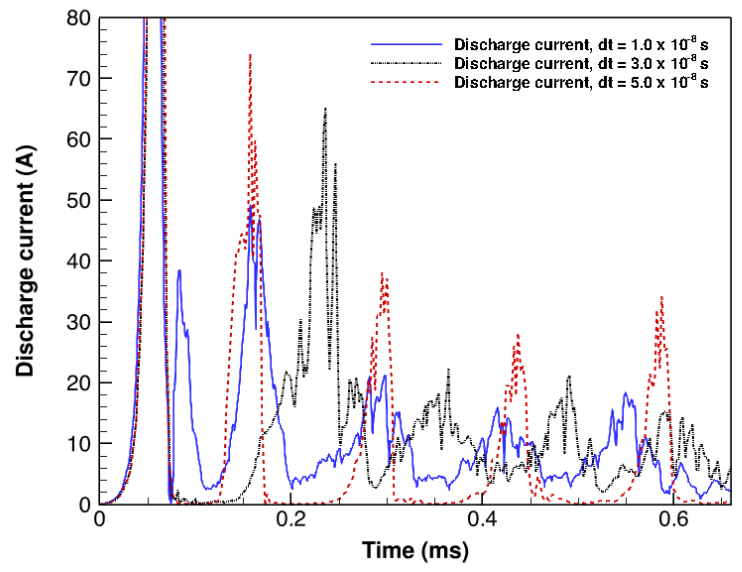

Figure 14. Discharge current vs time, different $\Delta t$.

\section{Conclusion}

Based on the computational results for the discharge current, instantaneous, and average plasma properties, the two-dimensional hybrid-DK simulation performs adequately when compared to Koo and Boyd's hybrid-PIC simulation. In the presence of a static electron mobility profile, the discharge current is dampened. To obtain a similar, nearly damped solution with the hybrid-PIC model, it is necessary to decrease the time step and increase the macroparticle count by a factor of approximately seven and thirty-six times, respectively. Even with a high fidelity hybrid-PIC simulation, it is still difficult to obtain convergence. Results indicate that both kinetic models are sensitive to boundary conditions in the fluid electron model, particularly at the anode. The damped discharge current in the DK case implies that a static electron mobility profile is insufficient for the dynamically changing plasma in a Hall thruster, but since these results may be coupled to an insuffient anode boundary condition, this implication must be studied more closely. As such, in the future, it will likely be necessary to install a dynamically changing mobility profile into the electron model and alter the boundary conditions to improve physical accuracy. 


\section{Acknowledgments}

The authors gratefully acknowledge financial support provided by Air Force Office of Scientific Research Grant No. FA95550-17-1-0035.

\section{References}

${ }^{1}$ Fife, J., Hybrid-PIC Modeling and Electrostatic Probe Survey of Hall Thrusters, Ph.D. Thesis, Massachusetts Institute of Technology, 1999.

${ }^{2}$ Koo, J.W., Hybrid PIC-MCC Computational Modeling of Hall Thrusters, Ph.D. Thesis, University of Michigan, 2005.

${ }^{3}$ Hagelaar, G.J.M., Bareilles,J., Garrigues, L., and Boeuf, J.P., "Two-dimensional model of a stationary plasma thruster," Journal of Applied Physics, Vol. 91, No. 9, May 2002, pp. 5592-5598.

${ }^{4}$ Scharfe, M.K., Gascon, N., Cappelli, M.A., and Fernandez, E., "Comparison of hybrid Hall thruster model to experimental measurements," Physics of Plasmas, Vol. 13, 2006, Article 083505.

${ }^{5}$ Hara, K., Development of Grid-Based Direct Kinetic Method and Hybrid Kinetic-Continuum Modeling of Hall Thruster Discharge Plasmas, Ph.D. Thesis, University of Michigan, 2015.

${ }^{6}$ Raisanen, A.L., Hara, K., and Boyd, I.D., "Comparing Two-Dimensional, Axisymmetric, Hybrid-Direct Kinetic and Hybrid Particle-in-Cell Simulations of the Discharge Plasma in a Hall Thruster," AIAA Paper 2016-4620, July 2016.

${ }^{7}$ Haas, J.M. and Gallimore, A.D., "Internal plasma potential profiles in a laboratory-model Hall thruster," Physics of Plasmas, Vol. 8, No. 2, 2001, Article 652.

${ }^{8}$ Peterson, P.Y., Gallimore, A.D., and Haas, J.M., "An experimental investigation of the internal magnetic field topography of an operating Hall thruster," Physics of Plasmas, Vol. 9, No. 10, 2002, Article 4364.

${ }^{9}$ Koo, J.W. and Boyd, I.D., "Modeling of Electron Mobility in Hall Thrusters," Physics of Plasmas, Vol. 13, 2006, Article 033501.

${ }^{10}$ Puech, V. and Mizzi, S., "Collision Cross Section and Tranport Parameters in Neon and Xenon," Journal of Physics D: Applied Physics, Vol. 24, 1991, pp. 1974-1985.

${ }^{11}$ Hara, K., Boyd, I.D., and Kolobov, V.I., "One-dimensional hybrid direct kinetic simulation of the discharge plasma in a Hall thruster," Physics of Plasmas, Vol. 19, 2012, Article 113508.

${ }^{12}$ Hara, K., Boyd, I.D., Sekerak, M.J., and Gallimore, A.D., "Breathing mode in Hall effect Thrusters," IEPC Paper 2015-283, July 2015.

${ }^{13}$ Lim, Y., Choe, W., Mazouffre, S., Park, J.S., Kim, H., Seon, J., and Garrigues, L., "Nonlinear ion dynamics in Hall thruster plasma source by ion transit-time instability," Plasma Sources Science and Technology, Vol. 26, No. 3, $2017,03 \mathrm{LT} 01$. 